\title{
Two major concerns surrounding governmental approval of tumourectomized kidney transplants in Japan
}

\author{
Akira Akabayashi ${ }^{1,2 *}$, Eisuke Nakazawa ${ }^{1}$ and Yuzaburo Uetake ${ }^{1}$ \\ ${ }^{1}$ Department of Biomedical Ethics, Faculty of Medicine, the University of Tokyo, Japan \\ ${ }^{2}$ Division of Medical Ethics, New York University School of Medicine, New York, USA
}

\section{Dear Editor,}

On July 6, 2018, the Japanese Ministry of Health, Labour and Welfare Expert Committee approved tumourectomized kidney transplant (TKT) as 'Advanced Medical Treatment' (AMT, 'senshiniryo'). In TKT, a kidney is removed as per a live donor operation and perfused with preservation fluid; the small renal tumour is excised on the back-table, and the tumourectomized kidney is transplanted into a recipient [1].

Alongside concerns expressed by experts and the media (e.g., risk management for cancerization and informed consent), we raise two more serious issues.

The first issue is the adequacy of hemodialysis (HD) compared to that of transplantation. TKT proponents emphasize the shorter life expectancy of those undergoing HD [2,3]. However, reliable empirical data supporting this assumption have not yet been obtained, due to difficulties in conducting unbiased RCTs to address this comparison. Patients with moderately serious conditions who can endure the invasive surgery and immunosuppressant therapy would likely benefit from TKT, while those with more serious conditions would likely fare better with $\mathrm{HD}$.

Regarding the age of recipients, an old analysis showed that patients who were 20 years to 39 years old at the time of placement on the waiting list, and who underwent transplantation, were projected to live longer than those who remained on the waiting list [4]. On the other hand, another study revealed that recipient age less than 50 years was a risk factor for early acute rejection episodes [5].

So far, no study has investigated how age directly impacts the survival difference between renal transplant and HD. However, analyses of Japanese databases identified some implications.

In 2016, the average age of 1,331 registered patients who underwent living kidney transplantation was 45.7 years $(22.3 \% \geqq 60)$. The 5 -year, 10 -year, and 15-year graft survival rates (data from 2000 to 2009) were $89.2 \%, 86.0 \%$, and $75.8 \%$, respectively [6].

In a sharp contrast, the average age of HD initiation was 69.4 years in roughly 39,000 patients $(77.0 \% \geqq 60)$, with a crude death rate of 9.7 . HD duration was $\geqq 5$ years in $50.4 \%$, $\geqq 10$ years in $25.2 \%$, and $\geqq 15$ years in $13.0 \%$. The total number of patients undergoing HD was roughly 330,000 in 2016 [7].

These data imply that older patients may benefit more from HD, while younger patients with less serious conditions may benefit more from transplantation. A rigorous analysis adjusting age is needed to answer this question, where transplantation patients are compared with HD patients under proper medical control.

Japan is in good standing with regard to HD [8], with very low mortality rates. Moreover, HD is relatively inexpensive, at JPY 4,400,000 (USD 40,000) annually, in sharp contrast to that in the United States (USD 82,000). These facts should also be considered in policy-making.

The more fundamental concern is the issue of allocating public resources to unestablished, non-life-saving treatment. Because HD is available as an alternative, TKT does not represent a life-saving measure as heart, lung, and liver transplantations would. One of the most significant benefits of TKT for HD patients is improved quality of life (QOL), with some reduction in medical expenditures, and the prevention of cardiovascular complications of HD patients.

Is it justifiable to use public national health insurance supported by taxpayers and employers' companies to cover TKT? A typical TKT surgery costs JPY 6,620,251 (USD 66,200). In the framework of AMT, patients would pay only JPY 1,127,385 (USD 11,300), while the remaining JPY 5,492,866 (USD 54,930) is covered by national health insurance.

It becomes exceedingly difficult to justify public spending on TKT. First, as a general principle, public funding requires accountability. How would the government explain using 'public' funding for an unestablished treatment for which the main benefit is improved QOL, without seeking other private sources such as pharmaceutical companies or private foundations?

Second, as TKT covers only a small fraction of the population, why are all Japanese citizens expected to contribute equally to increase the QOL of a few select patients? Is it not fundamentally unfair to require all to invest equally?

Third, the government did not hold any public Q\&A session on this matter, and even publicity for policy decision-making, a necessary condition of procedural justice, was not granted.

Therefore, we conclude that even the noblest of intentions for the best interests (increase in QOL) of patients secure no ethical justification to use public funds.

${ }^{*}$ Correspondence to: Akira Akabayashi, Department of Biomedical Ethics, Faculty of Medicine, the University of Tokyo, Japan, Fax: 81358413319; E-mail: akirasan-tky@umin.ac.jp

Received: August 24, 2018; Accepted: August 31, 2018; Published: September 03,2018 
Japan must face its paucity of brain-death organ donors, which has resulted in a lengthy waiting list. To this end, feasible recommendations have been made [9], but the government response has been underwhelming, with the petty promotion of a type of livingrelated organ transplantation (namely, TKT). Until dramatic changes are made in transplantation policies and the waiting list is shortened substantially, HD will remain the default treatment option for Japanese patients with end-stage renal failure.

\section{Competing interests}

Although AA is President of the Japan Association for Bioethics $(\mathrm{JAB})$, this paper reflects the authors' personal academic analyses and opinions. It does not represent JAB's official position on this issue. EN and YU have no conflicts of interest to declare.

\section{Funding}

None

\section{Authors Contribution}

AA: Conceptualization, Formal analysis, Writing-original draft, Writing-review \& editing.

EN: Conceptualization, Formal analysis, Writing - review \& editing.

YU: Conceptualization, Formal analysis, Writing - review \& editing.

\section{References}

1. Brook NR, Gibbons N, Johnson DW, Nicol DL (2010) Outcomes of transplants from patients with small renal tumours, live unrelated donors and dialysis wait-listed patients. Transplant International 23: 476-483.

2. Lugo-Baruqui A, Guerra G, Arocha A, Burke GW, Ciancio G (2016) Use of Kidneys with Small Renal Tumors for Transplantation. Curr Urol Rep 17: 3. [Crossref]

3. Musquera M, Perez M, Peri L, Esforzado N, Sebastià MC, et al. (2013) Kidneys from donors with incidental renal tumors: Should they be considered acceptable option for transplantation? Transplantation 95: 1129-1133.

4. Wolf RA, Ashby VB, Milford EL, Ojo AO, Ettenger RE, et al. (1999) Comparison of mortality in all patients on dialysis, patients on dialysis awaiting transplantation, and recipients of a first cadaveric transplant. New England J Medicine 341: 1725-1730.

5. Øien CM, Reisæter AV, Leivestad T, Dekker FW, Line PD, et al. (2007) Living Donor Kidney Transplantation: The Effects of Donor Age and Gender on Short- and LongTerm Outcomes. Transplantation 83: 600-606.

6. https://www.jstage.jst.go.jp/article/jst/52/2-3/52_113/_pdf/-char/ja

7. http://docs.jsdt.or.jp/overview/pdf2017/2016all.pdf

8. Robinson BM, Akazawa T, Jager KJ, Kerr PG, Saran R, et al. (2016) Factors affecting outcomes in patients reaching end-stage kidney disease worldwide: differences in access to renal replacement therapy, modality use, and haemodialysis practices. Lancet 388: 294-306.

9. Akabayashi A, Nakazawa E, Ozeki R, Tomiyama K, Mori K, et al. (2018) Twenty years after enactment of the Organ Transplant Law in Japan: Why are there still so few deceased donors? Transplantation Proceedings 50: 1209-1219.

Copyright: (C2018 Akabayashi A. This is an open-access article distributed under the terms of the Creative Commons Attribution License, which permits unrestricted use, distribution, and reproduction in any medium, provided the original author and source are credited. 